\title{
POWER-CONTROL FOR MULTIMODE TRANSCEIVERS
}

\author{
P. Cherriman, L. Hanzo \\ Dept. of Electr. and Comp. Sc., Univ. of Southampton, SO17 1BJ, UK. \\ Tel: +44-703-593 125, Fax: +44-703-593 045 \\ Email: pjc94r@ecs.soton.ac.uk, lh@ecs.soton.ac.uk \\ http://www-mobile.ecs.soton.ac.uk
}

\begin{abstract}
The performance of a power-control algorithm [7] suitable for multi-mode transceivers is investigated using 1,2 and $4 \mathrm{bit} / \mathrm{symbol}$ modems. It is shown that the algorithm is suitable for maintaining a target frame error rate, irrespective of the modulation mode employed. The transceiver parameters are summarised in Table 1, while the minimum and maximum required average transmitted powers in the cell for the different modes are given in Table 4.
\end{abstract}

\section{MOTIVATION}

Intelligent adaptive multi-mode transceivers or software radios [1],[3]-[6] are intensively studied under the auspices of a number of initiatives, such as for example the European Union's ACTS programme. In this programme we have been investigating adaptive modulation schemes [3,6], which can be re-configured to operate using different number of bits per modulation symbol, in order to exploit the timevariant channel capacity of fading channels. In order to benefit from the arising variable bitrate, we also investigated the feasibility of invoking the programmable-rate H.263 standard video codec and contrive adaptive packetisers, allowing us to design an integrated multimode video transceiver $[7,8]$. Furthermore, an efficient power-control scheme was required, which could support the operation of different modulation modes and the specific requirements imposed by the video codec.

\section{POWER CONTROL}

The multi-mode video transceiver and the power-control algorithm used were described in Reference [7], here only the rationale behind the scheme is stated. The transceiver parameters are summarised in Table 1 . The power-control technique is essentially a bit error rate (BER) based algorithm, which ensures a reliable channel quality estimation. This estimate is based on the binary $\mathrm{BCH}$ channel codec's error rate monitoring and over-load detection capability. The $\mathrm{BCH}$ codecs employed in the different modem modes were also summarised in Table 1. The algorithm reduces the transmitted power, if the number of transmission errors is well below the $\mathrm{BCH}$ codec's error correction capability and hence the $\mathrm{BCH}$ codec's error correction power is not fully exploited, while frequent $\mathrm{BCH}$ code over-load conditions indicate that the transmitted power has to be incremented. The power

VTC'97, PHOENIX, USA, MOBILE COMMUNICATIONS SESSION control step-size of the algorithm was also adjustable. For a detailed set of algorithmic parameters and a flow-chart the interested reader is referred to [7]. Let us now concentrate on the multi-mode performance of the algorithm in the next Section.

\section{PERFORMANCE OF MULTI-MODE POWER CONTROL}

The proposed multimode video transceiver of Table 1 using our power control algorithm was simulated and the worstcase scenario of a single interfer was employed in order to generate co-channel interference. The transmission frame error rate (FER) versus user distance and interferer distance from the base station (BS) is shown in Figure 1, when using 4-QAM and no power control. Since our H.263-based video transceiver exhibited a near-unimpaired perceptual video quality at a transmission FER of $5 \%$, we configured the power control scheme to maintain this target FER. Given the transceiver parameters used in this experiment, the FER is lower than $5 \%$ over most of the cell area, but it is above this threshold for the worst-case combinations of user and interferer distances, as demonstrated by the Figure. Observe also that when the interferer is at a distance of +200 $\mathrm{m}$ from its BS and hence the farthest from the serving BS, a maximum of $5 \%$ FER is maintained for all user distances within the cell. By contrast, for an interferer distance of $-200 \mathrm{~m}$, when the interferer is closest to the serving BS, the maximum acceptable user distance is about $140 \mathrm{~m}$. In other words, over the majority of the cell a better than required FER is maintained at the cost of a high transmitted power, while in certain cell areas the FER performance is inadequate.

In Figures 2(a) and 2(b) we displayed two operational scenarios for the best and worst case situations, in which the power-control scheme was used. BPSK mode was used and the best case was, when the interferer was as far from the serving BS, as possible, ie at $+200 \mathrm{~m}$ from its own BS, while the user was as close to its serving BSs, as possible, ie at 0 $\mathrm{m}$. By contrast, the worst case is when they are both at the edge of their cells, with the interferer in its own cell, but as close to the serving BS, as possible. Explicitly, Figures 2(a) and 2(b) display the power-controlled transmission power, slow fading envelope, the signal-to-interference+noise-ratio (SINR) averaged over a timeslot and the Frame Error Flag (FEF) as a function of time for the best and worst case interferer and user positions, respectively. Observe that in the best-case situation the transmitted power is close to its minimum of $-34 \mathrm{dBm}$, while the worst-case example requires a substantially increased transmitted power. The 


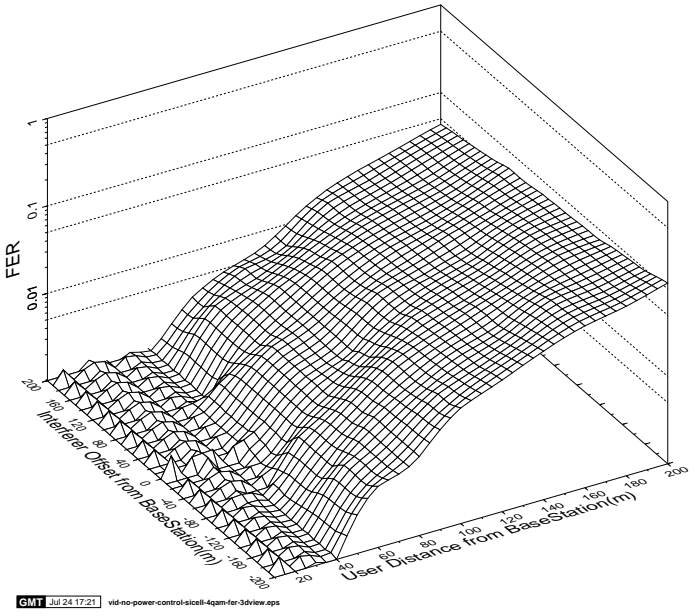

(a) 3D view

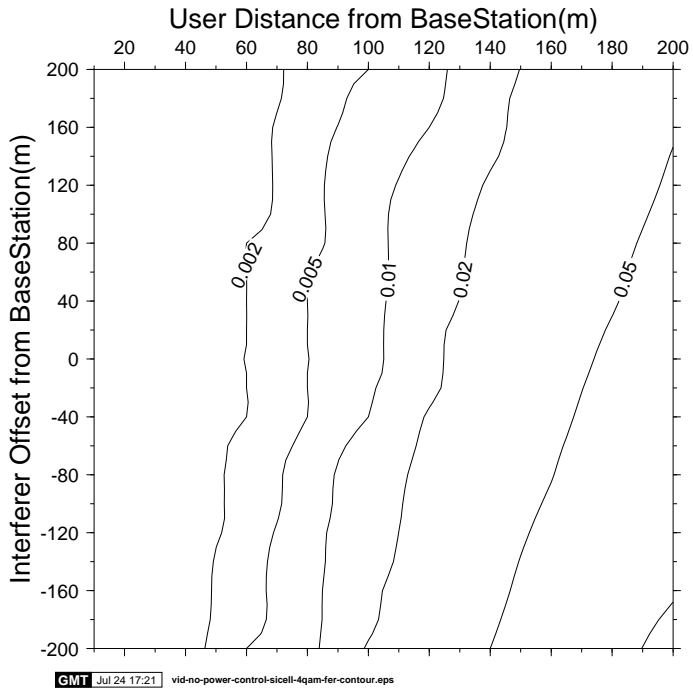

(b) Contour View

Figure 1: Frame error rate (FER) with no Power Control, versus user and interferer distance from basestation

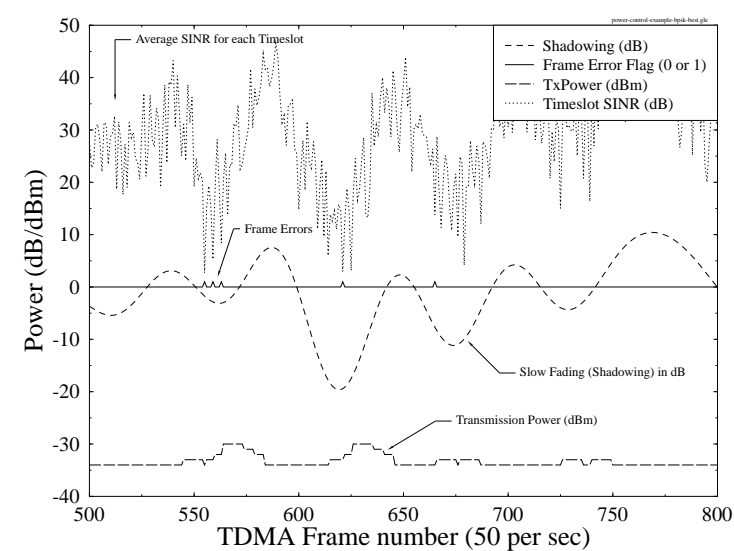

(a) Best Case

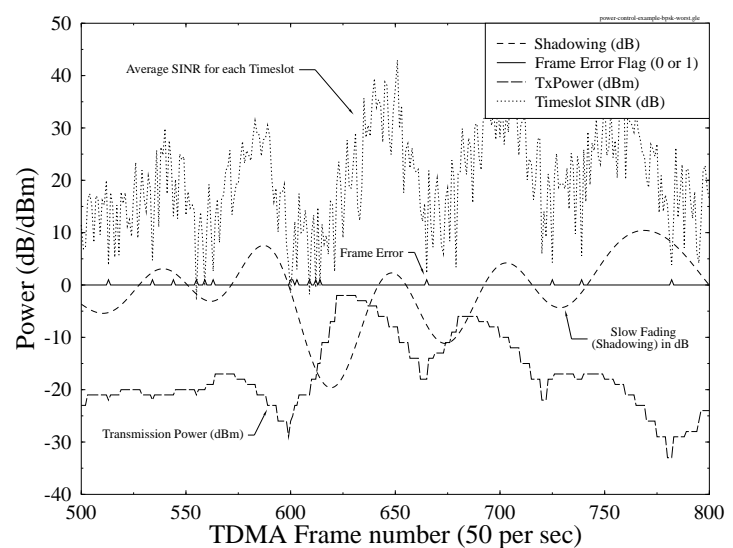

(b) Worst Case

Figure 2: Various waveforms associated with the error rate based Power Control Algorithm (a) Best case situation when both interfer and user are close to their basestation (b) Worst case situation, when both interfer and user are at the edge of their cell 


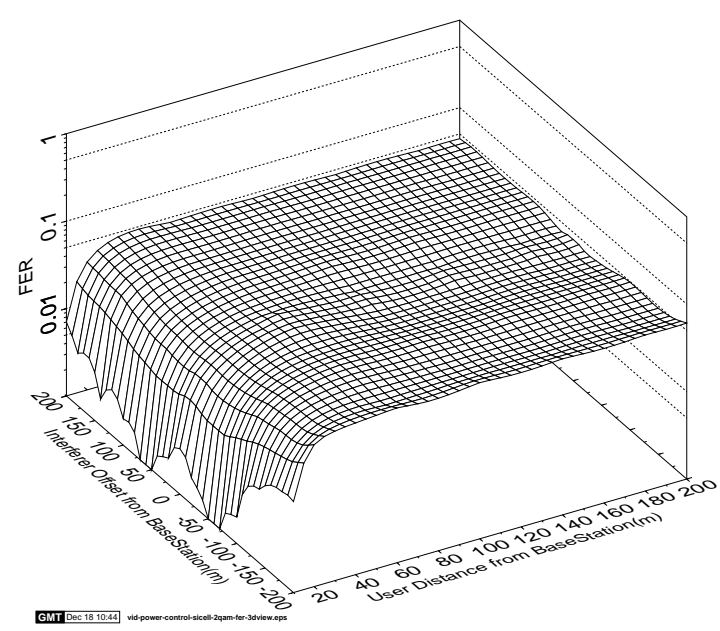

(a) BPSK

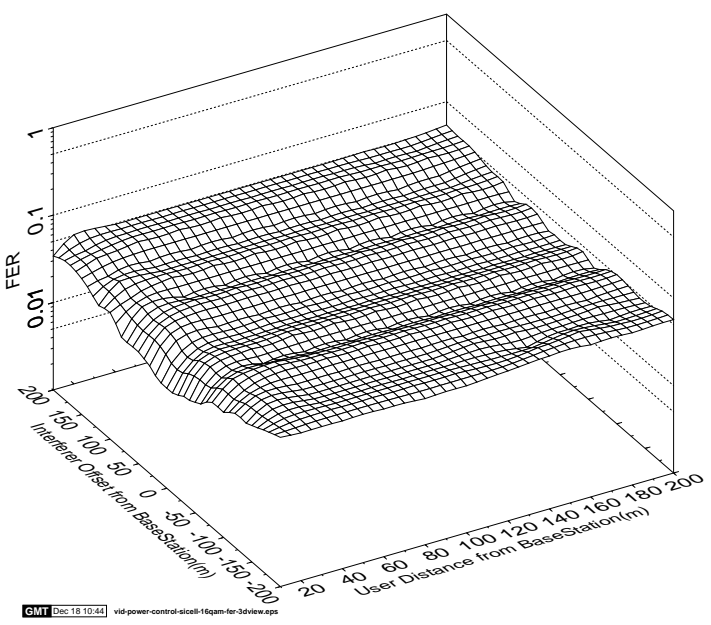

(b) $16 \mathrm{QAM}$

Figure 3: Frame error rate (FER) with Power Control, versus user and interferer distance from basestation

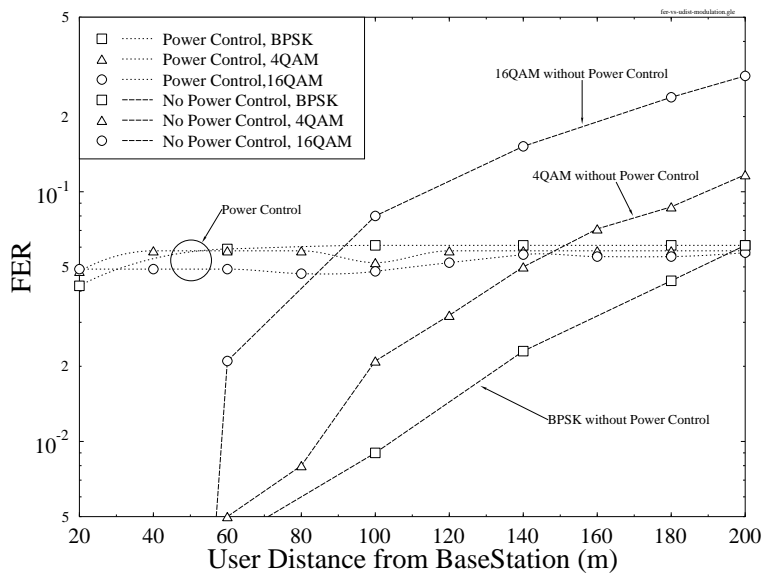

Figure 4: Frame error rate (FER) with and without Power Control, versus user distance from basestation, for the maximum interference that can be caused by a single interferer

worst-case example of Figure 2(b) requires a more accurate power control and demonstrates hence, how the power control attempts to compensate, after a one-frame latency, for degrading SINR values. Notice furthermore in the Figures how the FEF indicates, when for example a rapid power boost would be required to conpensate for a deep fade, but due to its inherent latency the scheme cannot respond sufficiently promptly.

Accordingly, as seen in Figure 3 for BPSK and 16QAM, the power control scheme achieves a near-constant transmission frame error rate over the geographic area of the cell.

\begin{tabular}{|l||l|l|l|}
\hline \multicolumn{1}{l||}{ Features } & \multicolumn{3}{c|}{ Multi-rate System } \\
\hline \hline Modem & PSA-BPSK & 4-PSAQAM & 16 -PSAQAM \\
\hline Bits/Symbol & 1 & 2 & 4 \\
\hline $\begin{array}{l}\text { No. of sub- } \\
\text { channels }\end{array}$ & 1 & 1 & 2 \\
\hline C1 FEC & BCH(127,85,6) & BCH $(255,171,11)$ & BCH(255,191,8) \\
\hline C2 FEC & N/A & N/A & BCH $(255,147,14)$ \\
\hline $\begin{array}{l}\text { Video-rate } \\
\text { (kbit } / \mathrm{s} \text { ) }\end{array}$ & 4.25 & 8.55 & 16.9 \\
\hline
\end{tabular}

\begin{tabular}{|l|c|}
\hline Features & General System \\
\hline \hline User Symbol Rate (kBd) & 7.3 \\
\hline No. of Users & 9 \\
\hline System Symbol Rate (kBd) & 131.4 \\
\hline System Bandwidth $(\mathrm{kHz})$ & 200 \\
\hline Eff. User Bandwidth $(\mathrm{kHz})$ & 11.1 \\
\hline TDMA frame length (ms) & 20 \\
\hline Slots/Frame & 18 \\
\hline Vehicular Speed & $13.4 \mathrm{~m} / \mathrm{s}$ or $30 \mathrm{mph}$ \\
\hline Propagation Frequency (GHz) & $6.2696 \times 10^{-4}$ \\
\hline $\begin{array}{l}\text { Fast Fading Normalised Doppler } \\
\text { Frequency }\end{array}$ & 6 \\
\hline $\begin{array}{l}\text { Log-Normal Shadowing standard devi- } \\
\text { ation (dB) }\end{array}$ & Power law 3.5 \\
\hline Pathloss Model & 1 \\
\hline Basestation Separation (km) & \\
\hline
\end{tabular}

Table 1: Summary of System features for the reconfigurable multimode mobile radio system

Observe in the Figures that in the extreme vicinity of the BS in the BPSK mode the power could not be reduced below the $30-64=-34 \mathrm{dBm}$ minimum level, which resulted in a reduced FER. In case of 16QAM, however, this phenomenon is less pronounced.

Figure 4 shows the system's FER performance for BPSK, 4QAM and 16QAM modes of operation. These curves were obtained by averaging the results of Figure 3 for various interferer distances. A conclusion of the above findings was that the proposed BER-based power-control algorithm was capable of assisting in different modulation schemes. 


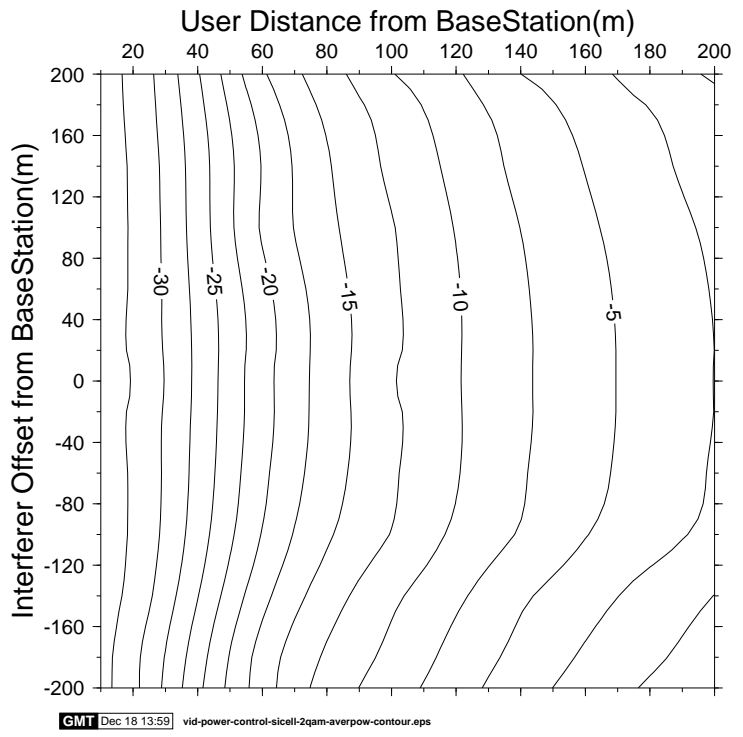

Figure 5: Average transmission power for a BPSK user, with a single BPSK interferer, versus user and interferer distance from basestation

However, when the vehicular speed or fading speed changes it has been found that parameter changes are needed in order to maintain the target FER performance. Although for BPSK the power control seem to actually degrade the FER performance, we will show that maintaining the required target FER allows the portables to operate at significantly reduced transmitted power and battery consumption.

\section{AVERAGE POWER}

When there is no power control, the average transmitted power is identical to the power of each portable. When using power control, the average transmitted power for a transceiver varies within the cell, and depends also on the modulation mode used. The corresponding simulation results are shown in Figures 6-7 for BPSK, 4QAM and 16QAM, respectively. Explicitly, these Figures demonstrate, how the average transmitted power varies with user and interferer distances from their respective basestations. As expected, the average required transmitted power increases, as users move away from their basestation, but the position of the interferer also has an effect. It should be noted that the maximum average power is less than $30 \mathrm{dBm}$, the power that we used for the fixed power simulations. We note furthermore that the position of the interfer has more of an effect in conjunction with the least robust 16QAM scheme, where the lowest transmitted power is required, when the interferers are close to their BS, transmitting also also low powers.

The average transmission power versus user distance from the BS at the best and worst interferer positions are plotted for BPSK, 4QAM, and 16QAM modes of operation in Figure 8. It was found from this Figure that the average power $P_{a v}$ in terms of user distance could be modelled using

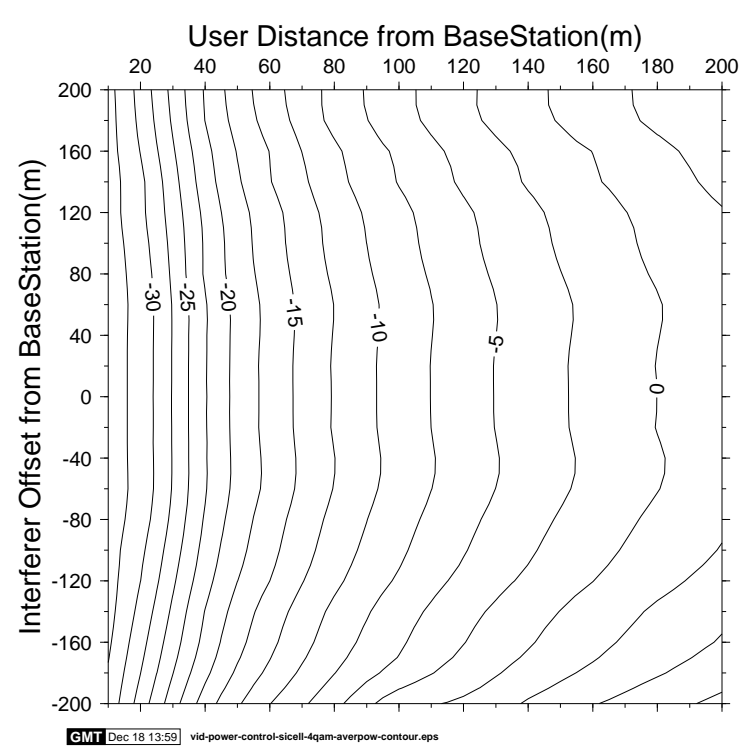

Figure 6: Average transmission power for a 4QAM user, with a single 4QAM interferer, versus user and interferer distance from basestation

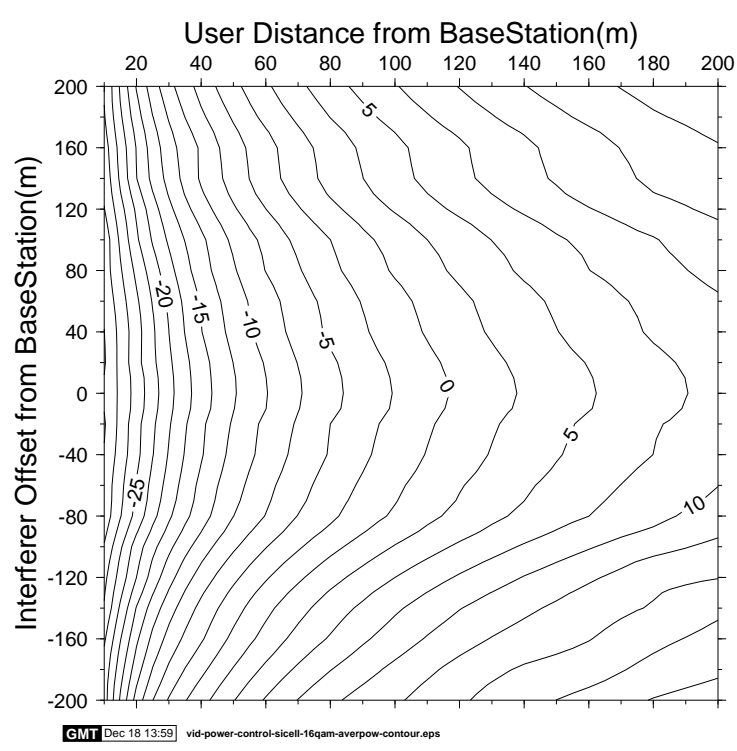

Figure 7: Average transmission power for a 16QAM user, with a single 16QAM interferer, versus user and interferer distance from basestation 


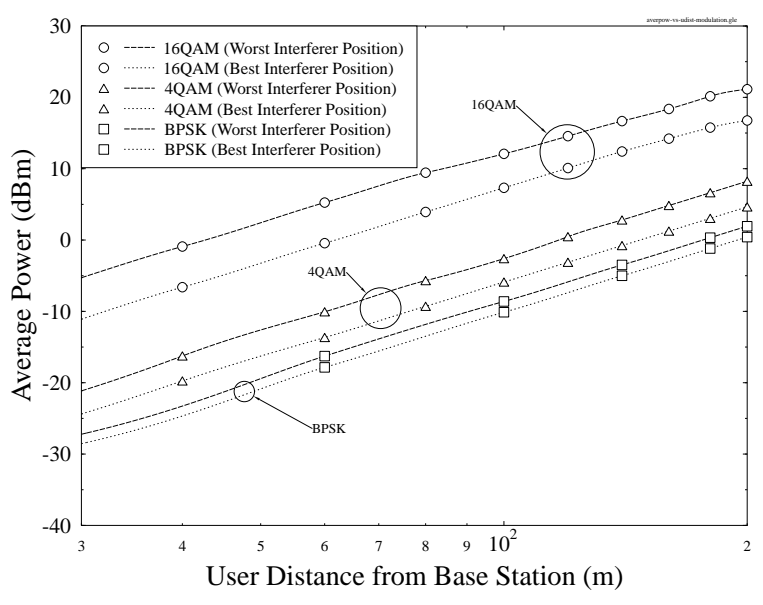

Figure 8: Average transmission power versus user distance from basestation, for the best $(200 \mathrm{~m})$ and worst $(-200 \mathrm{~m})$ interferer positions and for BPSK, 4QAM, and 16QAM modes of operation

Equation 1:

$$
P_{a v}(d B)=35 \log _{10}(d)+\beta,
$$

where $d$ is the user distance from the basestation and $\beta$ is an offset, dependent on the modulation scheme and interferer position. As expected, the $35 \cdot \log _{10}(d)$ term in the Equation is due to the inverse 3.5 power-law, which we used as our pathloss model.

The average power in the cell, assuming a uniform geographical portable distribution and a circular cell area, can be calculated using the mean value theoreom, stating that the average value for a two-dimensional function can be found by dividing the volume of the corresponding enclosed volume by the base area. In this case the base area is that of a circle, ie $\pi R^{2}$. The volume of the integral of Equation 1 for the range of possible user radii and angles from the basestation after division by $\pi R^{2}$ is given by:

$$
\begin{aligned}
\begin{array}{l}
\text { Aver. Power } \\
\text { in Cell }(\mathrm{dB})
\end{array} & =\frac{1}{\pi R^{2}} \int_{\phi=0}^{2 \pi} \int_{r=0}^{R}\left(35 \log _{10} r+\beta\right) r d r d \phi \\
& =35 \log _{10} R+\beta-\frac{35}{2 \times \ln 10} \\
& =35 \log _{10} R+\beta-7.6 d B .
\end{aligned}
$$

This Equation implies that the average transmission power in the cell is $7.6 \mathrm{~dB}$ less than the average power used by a user at the edge of the cell. In case of the 3.5 pathloss powerlaw model we used this is equivalent to the average power of a user at a distance of $0.61 \times R$ from his BS. Using the offset $\beta$ found from Figure 8 , the average transmission power in the cell is tabulated in Table 4 for the BPSK, 4QAM and 16QAM modes of our transceiver. Since $\beta$ is dependent on both the modulation scheme and the interferer position, as shown in Figure 8, the table includes results for the maximum and minimum average power that can be expected in a cell. The minimum average power occurs, when the interferer is close to its basestation, therefore transmits at a

\begin{tabular}{|c|c|c|}
\hline $\begin{array}{c}\text { Modulation } \\
\text { Scheme }\end{array}$ & $\begin{array}{c}\text { Minimum average } \\
\text { power in cell }(\mathrm{dBm})\end{array}$ & $\begin{array}{c}\text { Maximum average } \\
\text { power in cell }(\mathrm{dBm})\end{array}$ \\
\hline \hline BPSK & -9.82 & -5.67 \\
4QAM & -5.98 & 0.64 \\
16QAM & 0.68 & 15.02 \\
\hline
\end{tabular}

Table 2: Average Transmission Power in the cell in $\mathrm{dBm}$, for various modulation schemes and interferer positions

lower power, thereby reducing the amount of co-channel interference it inflicts. The maximum average power occurs, when the interferer is at the edge of its cell and at the closest point to the interfered BS.

In conclusion, it can be seen from the Table that even for the most demanding 16QAM mode the maximum required average power in the cell is about $15 \mathrm{~dB}$ less than $30 \mathrm{dBm}$. In comparison to the minimum required average power the transmitted power reduction is about $30 \mathrm{~dB}$. The simulations without power control used a fixed transmission power of $30 \mathrm{dBm}$, and achieved worst performance at the edge of the cells, as it was shown in Figure 4.

\section{ACKNOWLEDGEMENT}

The financial support of the EPSRC, UK in the framework of the contract GR/K74043 is gratefully acknowledged.

\section{REFERENCES}

[1] W. Webb and L. Hanzo, Modern Quadrature Amplitude Modulation: Principles and Applications for Wireless Communications. IEEE Press-Pentech Press, 1994.

[2] J. C. I. Chuang and N. R. Sollenberger, "Uplink power control for TDMA portable radio channels," IEEE Tr. on Vehicular Technology, vol. 43, pp. 33-39, Feb 1994.

[3] J. M. Torrance and L. Hanzo, "Upper bound performance of adaptive modulation in a slow Rayleigh fading channel," Electronics Letters, pp. 169-171, April 1996.

[4] T. Suzuki, S. Sampei, and N. Morinaga, "Space and path diversity combining technique for $10 \mathrm{Mbits} / \mathrm{s}$ adaptive modulation/TDMA in wireless communications systems," in Proceedings of IEEE VTC '96 pp. 10031007.

[5] S. Chua and A. Goldsmith, "Variable-rate variablepower mQAM for fading channels," in Proceedings of IEEE VTC' '96, pp. 815-819.

[6] J. M. Torrance and L. Hanzo, "Optimisation of switching levels for adaptive modulation in a slow rayleigh fading channel," Electronics Letters, pp. 1167-1169, June 1996.

[7] P. Cherriman, L. Hanzo: Power-controlled H.263 wireless videophone performance in iterference-limited scenarios, Proc. of Personal, Indoor and Mobile Radio Communications, PIMRC'96, Taipei, Taiwan, 1518 Oct., 1996, pp 963-967

[8] P. Cherriman, L. Hanzo: H261 and H263based Programable Video Transceivers, Proc. of ICCS'96/ISPAC'96 Singapore, Westin, 25-29. Nov. 1996, pp 1369-1373 